\title{
8 Kultur und somatoforme Störungen
}

\author{
Martin Aigner
}

Einleitend soll bei der Betrachtung des Zusammenhangs zwischen Kultur und somatoformen Störungen zunächst der Begriff „Kultur“ "kurz beleuchtet werden. Kultur befindet - und befand sich schon immer - in Veränderung und Bewegung mit den Menschen, die sie praktizieren. Kultur kann heute nicht mehr als ein einheitlicher Komplex von Sitten und Gebräuchen verstanden werden, der an ein bestimmtes Territorium, eine Region oder eine Nation gebunden ist, wie es in den klassischen Theorien der Ethnologie, Völker- oder Volkskunde üblich war. Kultur wird zwar weiterhin als sozial verbindendes Bedeutungssystem verstanden, allerdings muss Kultur in sich heterogener gedacht werden, als es bisher der Fall war. Der Begriff „Medicoscape“ scheint hier sehr brauchbar zu sein, um diesen flexiblen Kulturbegriff im medizinischen Bereich zu beschreiben. Unter Medicoscapes versteht man weltweit verstreute „Landschaften“ von Personen und Organisationen im heilkundlich-medizinischen Bereich, welche sich lokal verdichtet an einem Ort darstellen können, aber zugleich räumlich weit entfernte Orte, Personen und Organisationen miteinander verbinden. Zu einem Medicoscape zählen unter anderem international Therapiesuchende, Therapie offerierende Personen, weltweit agierende Pharmakonzerne, die Weltgesundheitsorganisation (WHO), traditioneller Heiler, regionale Heilpraktiken, global verbreitete Therapieformen - eben alle, die zu einem Medizinbereich beitragen und teilnehmen (Hörbst u. Krause 2004). Diese „Medizinlandschaften" haben bestimmte Begriffe, wie eben in dem vorliegenden Fall "somatoforme Störungen“, als „Kristallisationspunkte“ und überlappen bzw. grenzen sich ab gegenüber anderen Medicoscapes. Neben der Effizienz der Therapie entscheiden soziale Akzeptanz und der damit verbundene Umgang in der Gesellschaft über den „Erfolg“ oder „Misserfolg“ der verschiedenen Medicoscapes. 


\subsection{Kultureller Einfluss auf Symptom-Präsentation}

Im Medizinsystem neigen Patienten dazu, Symptome zu präsentieren, die „medizinisch richtig“ sind, d.h. Symptome, die Ärzte erwarten und verstehen. Somatische Symptome sind leichter zu erkennen/benennen und weniger stigmatisierend als psychische Symptome und können daher auch entsprechend leichter kommuniziert werden. Zudem versehen wir Gefühle mit „somatischen Markern“ (Birbaumer u. Schmidt 2006). Insbesondere dann, wenn Sprachbarrieren komplexe innerpsychische Konstrukte kaum in kurzer Zeit kommunizierbar machen, wird die Kommunikation über die körperliche Ebene verstärkt. In den meisten Kulturen wird psychosozialer Stress in körperliche Symptome „transformiert“ (Kleinman 1987). Dieser Prozess kann als Somatisierung beschrieben werden. Somatisierung kann also als ein Prozess gesehen werden, in dem die Patienten physische Symptome erleben, bei denen psychologischer Stress eine wesentliche Rolle spielt, die die Patienten jedoch hauptsächlich auf physische Ursachen zurückführen und daher im medizinischen Kontext medizinische Hilfe suchen. In der „WHO Cross-National Study“ von Gureje et al. (1997) wurde die Häufigkeit klinisch relevanter Somatisierung in 14 Ländern im Primärversorgungsbereich untersucht. Dabei wurden 5.438 Patienten mittels des Somatic Symptom Index (4/6) befragt, ob sie unter mindestens 4 - für Männer - bzw. mindestens 6 - für Frauen - körperlichen Symptomen leiden, für die es keine ausreichende medizinische Erklärung gibt. Im Mittel erreichten oder überschritten 19,7\% der Untersuchten diese Screeninggrenze. Die Bandbreite in den verschiedenen Ländern bewegte sich zwischen 7,6\% und 36,8\% der Befragten (Gureje et al. 1997). Diese Studie belegt eine gewisse Schwankungsbreite in der Häufigkeit der Somatisierung, die natürlich kulturabhängig sein dürfte. Grundsätzlich findet sich jedoch das Prinzip der Somatisierung in allen untersuchten Populationen. D.h. psychosozialer Stress ist mit körperlichen Symptomen verbunden, der Menschen dazu bringt, im Medizinsystem Hilfe zu suchen. Psychosozialer Stress erfordert eine Verhaltensänderung, um Stresssituationen durch adaptives Verhalten bewältigen zu können. Emotionen können als psychophysische Prozesse verstanden werden, die die Verhaltensbereitschaft verändern. Entsprechend sind Emotionen notwendig, um durch innerpsychische Signale und interpersonelle Kommunikation zur Verhaltensanpassung und Bewältigung der Stresssituationen führen zu können. Emotionen gehen als psychophysische Prozesse mit körperlichen („somatoformen“) Symptomen einher. Bei den physiologischen Emotionssystemen, wie dem Kampf-Flucht-System (Gray 1982) als in der Evolution herausgebildete Vorbereitung des Körpers für das Kampf-FluchtVerhalten, sind die körperlichen Symptome noch sehr uniform und kulturübergreifend. Mit der Zunahme der Komplexität des Verhaltensrepertoires haben sich die „Basisemotionen“ herausgebildet (z.B. die 7 Basisemotionen nach Paul Ekmann: Fröhlichkeit, Wut, Ekel, Furcht, Verachtung, Traurigkeit und Überraschung), die elementar und ebenfalls kulturübergreifend gefunden werden können. Eine weitere Komplexitätssteigerung unseres Verhaltens- 
repertoires erfordert schließlich sekundäre Emotionen bis hin zu Schemata, um „differenzierte Verhaltensbereitschaften“ zu ermöglichen, wie sie in einer komplexen modernen Gesellschaft notwendig sind. Kommt es bei den physiologischen Emotionsprozessen und Basisemotionen noch über die Kulturen hinweg zur Aktivierung ähnlicher neuronaler Netzwerke und damit auch ähnlicher körperlicher Symptome, so kommt es bei sekundären Emotionen bis zu den Schemata zu kulturell geformten „neuronalen Netzwerken“ und damit auch zu kulturell beeinflussten Symptombildungen. Die Gefühle (als subjektiver Anteil der Emotion) werden dabei mit „somatischen Markern“ (Birbaumer u. Schmidt 2006) wiedererkennbar gemacht. Transkulturelle Studien mit bildgebenden Verfahren haben nachgewiesen, dass der eigene kulturelle Hintergrund die neuronale Aktivität, die kognitiven Funktionen zugrunde liegt, beeinflussen kann. Die Ergebnisse ergeben einen neuartigen Ansatz durch die Unterscheidung von kultur-abhängigen und kultur-unabhängigen neuronalen Mechanismen der menschlichen Wahrnehmung (Han u. Northoff 2008) und damit auch kulturübergreifenden und kulturabhängigen Mustern körperlicher Symptome bei psychosozialem Stress, wobei natürlich keine Begriffsbildung „kulturunabhängig“ ist. Dennoch kann versucht werden kulturübergreifende Gemeinsamkeiten zu finden, wie dies in den globalen Diagnosesystemen z.B. des ICD-10 (WHO 1994) und DSM-IV (APA 1994) für die verschiedenen Diagnosekategorien wie z.B. für die somatoformen Störungen versucht wird. Darüber hinaus wird es notwendig sein, auch auf kulturelle Besonderheiten einzugehen, wie dies in Form der „culture bound syndromes“ in den Diagnosesystemen ebenfalls der Fall ist.

\subsection{Kulturgebundene Syndrome („culture bound syndromes“)}

Ob kulturgebundene Syndrome tatsächlich „existieren“, ist innerhalb der evidenzbasierten Medizin umstritten. Vor allem zwischen Anthropologen und Psychiatern wird die Frage kontrovers diskutiert. Diese Diskussion ist jedoch nur eine Frage des Standpunktes: Im Rahmen der Anthropologie werden kulturspezifische Aspekte besonders betont. Im Rahmen der Psychiatrie werden eher universelle neuropsychologische Erklärungsansätze gesucht. Beide Ansätze können jedoch in der transkulturellen Psychiatrie gewinnbringend verbunden werden. Der Ansatz, universelle Modelle für Krankheiten zu entwickeln, ermöglicht zum Beispiel den Einsatz von modernen Therapiestrategien wie Psychopharmaka, aber natürlich auch evidenzbasierter Verfahren wie Psycho- oder Physiotherapie. Andererseits sollten in einem ressourcenfördernden psychotherapeutischen Setting die jeweiligen kulturellen Ressourcen für eine Symptom- und Krankheitsbewältigung nicht unterschätzt werden. So ist es weniger wichtig, ob diese Syndrome „existieren“ oder nicht, sondern ob man mittels transkultureller Kompetenz und „globalisierter“ Diagnostik beide Ansätze für eine gelungene Therapie, für eine gelungene Bewältigung, verbinden kann. 
Kulturgebundene Syndrome werden kulturintern als echte Krankheit eingestuft. Sie haben innerhalb der jeweiligen Kultur einen großen Bekanntheitsgrad. In anderen Kulturen hingegen „fehlt die Kenntnis“ über diese Krankheit, es gibt keine nachweisbaren biochemischen oder organischen Ursachen und die Diagnose sowie die Therapie erfolgen meist innerhalb der lokalen Volksmedizin. Einige kulturgebundene Syndrome können körperliche Symptome (z.B. Schmerzen, funktionelle Körperstörungen) aufweisen, während andere sich ausschließlich in Verhaltensstörungen äußern. Wird diese Definition mit der Definition der somatoformen Störungen verknüpft, ergeben sich die kulturgebundenen „somatoformen“ Störungen. Zentrale Punkte dieser „Störungsgruppe“ sind demnach:

- psychische und/oder somatische Symptome, die auf eine bestimmte Gesellschaft oder Kultur beschränkt sind

- biochemische Ursachen oder Organveränderungen sind dabei nicht nachweisbar

Der Punkt „in anderen Kulturen ist das Krankheitsbild unbekannt“ bzw. „auf eine bestimmte Gesellschaft oder Kultur beschränkt" kann in einer globalisierten Informationsgesellschaft kaum aufrechterhalten werden. Ein Medicoscape einer bestimmten Störung lässt sich eben nicht auf eine bestimmte Menschengruppe beschränken, sondern unterschiedliche Menschen nehmen unterschiedlich intensiv daran teil oder grenzen sich unterschiedlich stark davon ab.

\subsection{Kulturgebundene „somatoforme“ Störungen}

Im Folgenden sollen einige kulturgebundene „somatoforme“ Störungen näher beschrieben werden. Der Begriff „somatoform“ ist dabei allerdings als problematisch anzusehen und sollte durch den Begriff „somatisch“ bzw. „körperlich" ersetzt werden. Wie oben bereits diskutiert, sind Emotionen eben psychophysische Prozesse, die psychische und physische Anteile vereinen. Die körperlichen Symptome sind daher „echt“ und sehen nicht nur so aus wie körperliche Symptome. Die Krankheitswertigkeit und Bedeutung der Symptome kann allerdings unterschiedlich gesehen werden. Bei den „somatoformen Störungen " wird das körperliche Symptom von den Patienten typischerweise katastrophisiert und im medizinischen Kontext um eine entsprechende Hilfe gesucht - eben „somatisiert“. Je nachdem, welche Symptome bzw. Symptomkomplexe besonders beachtenswert für die Teilnehmer an einem Medicoscape sind, kommt es zur Herausbildung von Diagnosebegriffen, die als kulturgebundene „somatoforme“ Symptome gesehen werden können:

Dhat (in Indien [jiryan], in Sri Lanka [sukra prameha], in China [shen-k'uei]) kann als eine kulturgebundene somatoforme Störung angesehen werden, mit hypochondrischen Ängsten und Sorgen, die sich auf den Verlust von Sperma 
beziehen, die weißliche Verfärbung des Urins sowie das Gefühl von Schwäche und Erschöpfung. Stehen die hypochondrischen Ängste im Vordergrund könnte das Syndrom als Hypochondrie klassifiziert werden. Sind die körperlichen Beschwerden im Vordergrund, dann könnte zum Beispiel nach ICD-10 (WHO 1994) eine autonome somatoforme Funktionsstörung diagnostiziert werden, wenn sich die Beschwerden nur auf das Genitalsystem beziehen, oder eine Neurasthenie, wenn die Schwächegefühle das klinische Bild dominieren. Da natürlich das Grundkonzept der Störung die Wertigkeit der Symptome entscheidend mitbestimmt, haben kulturgebundene Erklärungen eine wichtige Bedeutung.

Koro (malaiisch; etymologisch strittig, vielleicht „schrumpfend“ oder „Schildkröte[nkopf]“) beschreibt eine in Indonesien und Malaysia vorkommende Störung. In China wird diese Suo yang (Mandarin) oder Shuk yang, $\mathrm{S}(\mathrm{h})$ ook yong (Kantonesisch) („,schrumpfender Penis“) genannt. Im Westen wird übergreifend vom Syndrom der genitalen Retraktion (SGR) oder "genital retraction syndrome“ gesprochen. Die Störung besteht in der hypochondrischen Angst, dass der eigene Penis schrumpfe oder sich in den eigenen Körper zurückziehe und man davon sterbe. Bei Frauen bezieht sich die Angst natürlich auf die weiblichen Geschlechtsorgane. Nach einer Überblicksarbeit der nicht epidemischen Koro-Fallberichte zwischen 1954 bis 2005 von Garlipp (2008) sind die Fallberichte jedoch nicht auf den asiatischen Raum beschränkt, sondern können praktisch über die ganze Welt verstreut gefunden werden, also quasi ein Medicoscape „Koro“.

\subsection{Brain-Fag, Chronic Fatigue Syndrome}

Brain-Fag wurde bei Westafrikanischen Studenten in den 196oer „gefunden“. Doch der Begriff „brain fag“ taucht schon 1852 in USA auf, um ein Überlastungssyndrom zu charakterisieren (,überlastetes Gehirn“) und 1877 als „psychische Erschöpfung “ähnlich wie die Neurasthenie oder das „chronic fatigue syndrom“ (CFS). CFS ist durch schwere chronische Müdigkeit für mindestens sechs Monate oder länger charakterisiert und für die Diagnose müssen 4 oder mehr der folgenden Symptome auftreten:

- nach Belastung Gedächtnis- oder Konzentrationsstörungen

- nicht erholsamer Schlaf

- Muskelschmerzen

- Multi-Gelenkschmerzen

- empfindliche zervikale oder axilläre Lymphknoten

- Halsschmerzen

- Kopfschmerzen 


\subsection{Fibromyalgie}

Das Fibromyalgie-Syndrom (FMS) (engl.: fibromyalgi; überlappende Begriffe und Synonyme: Fibrositis, Myitis chronica, Myofaszitis, Myodysneuria, rheumatische Myositis, noduläre Fibromyositis, fibropathisches Syndrom, muskulärer Rheumatismus, ...) wurde 1990 im westlichen medizinischen Kontext durch das American College of Rheumatology (ACR) genauer definiert: Es ist gekennzeichnet durch Muskelschmerzen der oberen und unteren Extremitäten sowie der rechten und linken Körperhälfte, der Wirbelsäule und der vorderen Thoraxwand über 3 Monate, wobei mindestens 11 von 18 Druckpunkten bei digitaler Palpation schmerzhaft sein müssen und dabei die Laborbefunde unauffällig sein sollen (Wolfe et al. 1990). Das FMS wird traditionellerweise nicht unter den „culture bound syndromes“ angeführt, jedoch kann dieser Begriff in der globalisierten Welt auch als Kristallisationspunkt der „Medicoscape Fibromyalgie“ gesehen werden, weil es viele kulturgebundene Aspekte aufweist und es auch immer wieder Ärzte gibt, die sich diesem Begriff bzw. Medicoscape nicht anschließen und ihn infrage stellen. Es finden sich in der Definition im Wesentlichen subjektive Kriterien, die als Kommunikationsform von Schmerz- und Leidenserleben zwischen Patienten und Ärzten gesehen werden können. Es geht dabei stark um einen Erklärungsversuch von körperlichen Symptomen und auch einen Bewältigungsversuch bzw. um den Appell um Unterstützung (siehe dazu auch rationale Schmerzbewältigung). Diese Kriterien wurden für wissenschaftliche Zwecke entwickelt. Die Nützlichkeit für die Primärversorgung ist jedoch wie bereits erwähnt umstritten. In den deutschen AMWF-Leitlinien „Fibromyalgie“ werden diese Druckpunkte für die klinische Routinediagnostik als fakultativ angesehen. Der Schwerpunkt der Definition liegt auf „chronic widespread pain“(CWP), also chronische Ganzkörperschmerzen (Themenheft Fibromyalgie 2008). Aus psychiatrischer Sicht ergibt sich eine Überlappung zur „anhaltenden somatoformen Schmerzstörung“, bei der nach ICD-1o ein chronischer Schmerz über mindestens 6 Monate bestehen muss, keine ausreichende medizinische Erklärung gefunden werden kann und psychosoziale Faktoren in der Verstärkung sowie Aufrechterhaltung der Ganzkörperschmerzen beteiligt sind.

\subsection{Kulturelle Modelle und Symptomerleben: von Psychosomatik zu Soziosomatik}

Kulturelle Einflüsse auf Symptomerleben und Symptomkommunikation finden auf mehreren Ebenen statt: Psychophysiologie, Aufmerksamkeit, Symptomattribuierung und -interpretation, Art der Bewältigung, Hilfesuche und Behandlung. Somatische Symptome haben unterschiedliche psychologische und soziale Bedeutungen. Somatische Symptome können gesehen werden als (Kirmayer 2008): 
- Index für eine körperliche Erkrankung

- Ausdruck einer Psychopathologie (somatoform)

- Symbol für einen intrapsychischen Konflikt

- kulturell-kodierter Ausdruck für Disstress

- Medium für den Ausdruck von sozialer Unzufriedenheit

- Mechanismus, durch welchen Patienten versuchen, sich in ihrer Welt neu $\mathrm{zu}$ positionieren

Viele Patienten mit somatischen kulturellen Idiomen der Not beschreiben die sozialen Probleme, die ihre Symptome verstärken, wenn sie ein offenes Ohr finden. In der biomedizinischen Gesundheitsfürsorge spielen sie die sozialen Dimensionen herunter, weil sie Stigmatisierung fürchten. Dabei darf nicht vergessen werden, dass diagnostische Systeme selbst auch kulturelle Artefakte sind. Neben der Anleitung zur Therapie dienen diagnostische Etikettierungen auch der Zuschreibung von berechtigtem Leiden, deuten auf die Ernsthaftigkeit und Bedeutung für andere hin und haben sozialen Folgen wie Berechtigung für Krankenstand, Berentung etc. Bei Fehlen einer klaren Diagnose und der dadurch fehlenden effektiven Behandlung der Patienten kommt es zur Unsicherheit und zur Bemühung um eine definitive Diagnose, um eine Legitimation für das Leiden zu finden und eine Bewältigung des Leidens $z u$ erreichen. Was teilweise dann als Psychopathologie gesehen werden kann, kann auch auf diese soziale Notlage zurückgeführt werden. Nach Kohnen (2003) können unterschiedliche Krankheitsbewältigungsstrategien unterschieden werden, die einen hohen Grad an „Kulturgebundenheit“ aufweisen. Unter anderem unterscheidet Kohnen religiöse, willentliche, familiäre und rationale Krankheitsbewältigung:

- Bei der religiösen Krankheitsbewältigung führt Kohnen die Leitüberzeugung an: „Nur Gott hilft bei der Lebens- und Schmerzbewältigung wirklich!“ Das Verhalten bei körperlichen Beschwerden/Schmerzen kann dahingehend beschrieben werden, dass körperliche Beschwerden/Schmerz ertragen und erduldet werden, damit es als „Zeichen und die Botschaft Gottes“ erkannt werden kann. Die Handlungsanweisung, die daraus abgeleitet wird, lautet: „Körperliche Beschwerden/Schmerz enthalten einen besonderen Sinn, der zum Nachdenken über das eigene Leben anregt.“

- Bei der willentlichen Krankheitsbewältigung ist die Leitüberzeugung ganz ähnlich dazu: „Ich werde körperliche Beschwerden/Schmerzen nicht zulassen und falls es doch dazu kommt, diese unterdrücken. Folglich werde ich mich in die Einsamkeit zurückziehen und körperliche Beschwerden/Schmerzen ertragen." Die Kontrollüberzeugung jedoch unterscheidet sich schon stark: „Ich alleine werde mit meinem Willen körperliche Beschwerden/Schmerz bewältigen. “ Dadurch nimmt die Selbstkontrolle zu (Kompetenzvertrauen, self-efficacy [Bandura 1978]) und das Erleben von Vorhersagbarkeit und Kontrollierbarkeit wird als entängstigend erlebt. Bei dieser Bewältigungsform ist es „unfein, körperliche Beschwerden/Schmerz zu äußern“. 
- Ganz anders bei der familiären Krankheitsbewältigung: Die körperlichen Beschwerden/Schmerzen werden den anderen deutlich präsentiert, gemäß der Leitüberzeugung: „Die Familie hilft bei der Lebens- und Schmerzbewältigung durch familiäre Unterstützung und soziale Zuwendung. Nur wer seine Hilfsbedürftigkeit (deutlich) äußert, dem kann geholfen werden. “ Körperliche Beschwerden/Schmerzen werden also „zugelassen" und deutlich gegenüber anderen geäußert. Im Mittelmeerraum, bei christlichen wie auch bei den moslemischen Cruppen, ist die familiäre Bewältigungsstrategie weit verbreitet.

- Die rationale Krankheitsbewältigung passt gut zum biomedizinischen System. Gemäß der Kontrollüberzeugung: „Wenn ich meine körperlichen Beschwerden/Schmerz so präzise wie möglich beobachte und dem Arzt beschreibe, dann kann er am sichersten die Lokalisation der Cesundheitsstörung herausfinden, die Krankheit diagnostizieren und eine ursächliche Therapie einleiten. "Die Patienten sind dabei überzeugt, dass körperliche Beschwerden/Schmerzen technisch und fachlich bewältigt werden sollen. Daher werden die körperliche Beschwerden/Schmerzen nüchtern und möglichst ohne emotionale Beteiligung „quasiobjektiv“ beobachtet und geschildert. Dabei ist sofortiges Handeln und Einholen einer fachlichen Beratung besonders wichtig (Kohnen 2003).

\subsection{Somatoforme Störung: ein „kulturelles“ Konstrukt}

Somatoforme Störungen können so als ein „kulturelles Konstrukt“ gesehen werden, bei dem die Kommunikation von Krankheiten/Stress und die Bewältigung von Krankheiten/Stress in einer globalisierten Welt im Zentrum stehen. Dabei spielen kulturelle Modelle von somatischen Symptomen eine wichtige Rolle:

- Erklärungsmodelle, die Kausalattributionen zulassen und auf spezifische Mechanismen oder pathophysiologische Prozesse hindeuten.

- Prototypen, markante Bilder oder Vorbilder aus der eigenen Erfahrung, der Familie, dem Freundeskreis, aus den Massenmedien und der populären Kultur, die ein Reflektieren über den eigenen Zustand ermöglichen.

- implizite Modelle und prozedurales Wissen, das schwierig artikuliert werden kann, weil es in körperliche Abläufe, Stress zu erfahren, eingebettet ist, was zu spezifischen Verhaltens- und Kommunikationsweisen führt.

Für das DSM-V wird die Komplexe Somatische Symptom Störung („Complex Somatic Symptom Disorder“) vorgeschlagen, die übergreifende Charakteristika der somatoformen Störungen zusammenfasst und diese Diagnosen in Zukunft ersetzen soll:

1. Multiple somatische Symptome oder ein schweres Symptom, welche Leiden verursachen.

2. Fehlattribution bzw. übermäßige Sorgen oder Beschäftigung mit somatischen Symptomen und Krankheit (starke Krankheitsängstlichkeit, 
normale Körpersymptome werden als bedrohlich bewertet, katastrophisieren).

3. Dauer des Symptomzustandes (,state of being symptomatic“) über mindestens 6 Monate, wobei das einzelne Symptom nicht ständig präsent sein muss.

\section{Zusammenfassung}

Somatoforme Störungen können als ein „kulturelles“ Konstrukt mit „speziellen kulturellen Ausprägungen“ sowie „kulturübergreifenden Prinzipien“ verstanden werden: Kommunikation von Krankheiten/Stress in verschiedenen Kulturen ist für die Diagnostik und Modellbildung (Klärung) wichtig, ebenso das „Abholen der Patienten“ bei ihren individuellen Störungsbildern (kultureller Hintergrund, Medicoscape) und die Zuordnung zu „allgemeingültigen Störungsbildern“. Auch bei der Bewältigung von Krankheiten/Stress kann die Verbindung beider Ansätze nützlich sein: das Nützen therapeutischer Möglichkeiten allgemeingültiger Störungsbilder und das Nützen individueller Bewältigungsressourcen in verschiedenen kulturellen Kontexten.

Dieser Beitrag basiert auf einem Artikel des Autors in Spectrum Psychiatrie (Aigner 2011). Mit freundlicher Genehmigung der MedMedia Verlag und Mediaservice $\mathrm{GmbH}$.

\section{Literatur}

Aigner M (2011) Kultur und somatoforme Störungen. Spectrum Psychiatrie 1

American Psychiatric Association (1994) Diagnostic and Statistical Manual of Mental Disorders. 4. Auflage. APA Washington $D C$

Birbaumer N, Schmidt RF (2006) Biologische Psychologie. 4. Auflage. Springer Berlin Heidelberg New York

Gray IA (1982) The neuropsychology of anxiety: An inquiry into the functions of the septo-hippocampal system. Oxford University Press Oxford

Gureje 0, Simon GE, Ustun TB, Goldberg DP (1997) Somatization in cross-cultural perspective: a World Health Organization study in primary care. Am I Psychiatry 154,989-95

Han S, Northoff G (2008) Culture-sensitive neural substrates of human cognition: a transcultural neuroimaging approach Nature Reviews Neuroscience 9, 646-654

Hörbst V, Krause K (2004) Die Globalisierungsdebatte in der Medizinethnologie. Curare 27, 41-60

Kirmayer L) (2008) Culture and the metaphoric mediation of pain. Transcult Psychiatry 45, 318-38

Kleinman A (1987) Anthropology and psychiatry: The role of culture in cross-culture on illness. Br. J. Psychiatry 151, $447-454$

Kohnen N (2003) Von der Schmerzlichkeit des Schmerzerlebens. Wie fremde Kulturen Schmerzen wahrnehmen, erleben und bewältigen. 1. Auflage. pvv Ratingen

Themenheft Fibromyalgie (2008) Interdisziplinäre S3-Leitlinie. Definition, Pathophysiologie, Diagnostik und Therapie des Fibromyalgiesyndroms. Der Schmerz 22 Themenheft

WHO (1994) International Classification of Diseases (ICD). 10. Kapitel F

Wolfe F, Smythe HA, Yunus MB, Bennett RM, Bombardier C, Goldenberg DL, Tugwell P, Campbell SM, Abeles M, Clark P, Fam, AG, Farber SI, Fiechtner II, Franklin CM, Gatter, RA, Hamaty D, Lessard J, Lichtbroun AS, Masi AT, Mccain GA, Reynolds W], Romano TJ, Russel II, Sheon RP (1990) The American College of Rheumatology criteria for the classification of fibromyalgia: report of the multicenter criteria committee. Arthritis Rheum 33, 160-172 